Notre Dame Journal of Formal Logic Volume XV, Number 1, January 1974 NDJFAM

\title{
A NOTE ON NATURAL DEDUCTION IN MANY-VALUED LOGIC
}

\author{
BRUCE WHITE
}

Natural-deduction techniques have not been applied very much in the formalization of many-valued logics, since the deduction theorem fails for many interesting systems; this point is made, for example, by Ackermann [1]. Nevertheless, natural-deduction formalizations are possible-an interesting example is Woodruff's [3]. In this note I describe a very simple natural-deduction system $Q$ with rules in the style of Suppes [2]. The theorems of $\mathbf{Q}$ coincide with the theorems of $\mathbf{P}$, which are the consequences under modus ponens of the axiom schemes

A1 $A \rightarrow(B \rightarrow A)$

A2 $(A \rightarrow B) \rightarrow((B \rightarrow C) \rightarrow(A \rightarrow C))$

A3 $A \rightarrow((A \rightarrow B) \rightarrow B)$

In $Q$ the items in proofs are pairs $m A$ where $m$ is a set, possibly empty, of positive integers and $A$ is a formula. The numbers in $m$ indicate the assumptions upon which $A$ depends. The rules of $Q$ are

R1 For any formula $A$, the pair (i) $A$ may be introduced at step number $i$ in a proof.

R2 If $m$ and $n$ are disjoint, $k B$ may be inferred from $m A$ and $n(A \rightarrow B), k$ being the union of $m$ and $n$.

R3 From (i) A occurring at step $i$ and $m B$ one may infer $k(A \rightarrow B)$, where $k$ is the result of removing $i$ from $m$.

$A$ is a theorem of $Q$ if $\phi A$ is provable. $\varnothing$ is the null set. A1-A3 are easily shown to be theorems of $Q$ :

1. (1) $A \quad \mathrm{R} 1$

2. (2) $B \quad \mathrm{R} 1$

3. (1) $B \rightarrow A \quad 1,2, \mathrm{R} 3$

4. $\varnothing A \rightarrow(B \rightarrow A) \quad 1,3, \mathrm{R} 3$

Henceforth we omit $\varnothing$.

1. (1) $A \rightarrow B \quad \mathrm{R} 1$

2. (2) $B \rightarrow C \quad \mathrm{R} 1$ 


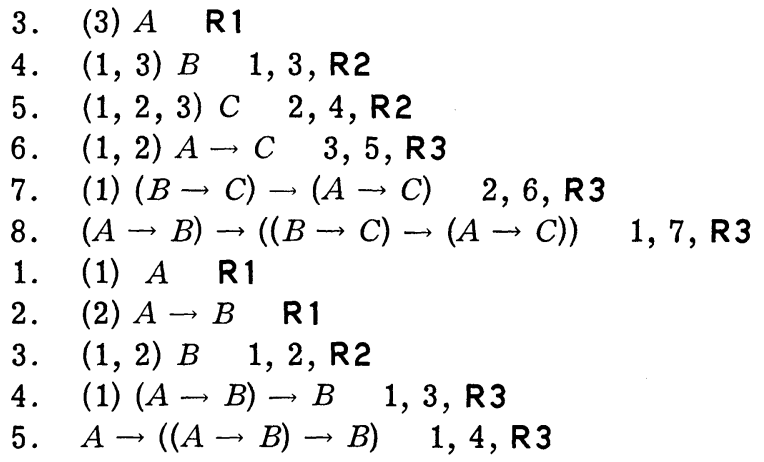

The class of theorems of $Q$ is closed under modus ponens, since $\varnothing$ is disjoint from $\varnothing$. $Q$ has the nice feature that not only is it easy to construct proofs in the system but it is also easy to see what cannot be proved in it. For example, any attempt to prove $(A \rightarrow(A \rightarrow B)) \rightarrow(A \rightarrow B)$ is blocked by the disjointness provision in $\mathrm{R} 2$.

To see that every theorem of $\mathbf{Q}$ is a theorem of $\mathbf{P}$ one gives an inductive proof that if $m A$ occurs in a proof of $\mathrm{Q}$ then $A_{m}^{*}$ is a theorem of P. $A_{m}^{*}$ is $A$ if $m$ is empty, otherwise it is the implication of $A$ by the assumptions whose numbers are in $m$. Thus if $m$ is $(1,2,3)$ and the $i$ 'th assumption is $B_{i}$, then $A_{m}^{*}$ is $B_{1} \rightarrow\left(B_{2} \rightarrow\left(B_{3} \rightarrow A\right)\right)$.

The formal proof of this will be omitted. Instead I shall give an example. Suppose $(1,2,3) B$ occurs in a proof as a result of (1) $A$ and $(2,3) A \rightarrow B$. Suppose $B_{1}$ is $C, B_{2}$ is $C, B_{3}$ is $D$ and we know that (a) $C \rightarrow A$ and (b) $C \rightarrow(D \rightarrow(A \rightarrow B))$ are theorems of $\mathrm{P}$. The following are wellknown theorems of $\mathbf{P}$ :

(c) $(A \rightarrow(B \rightarrow C)) \rightarrow(B \rightarrow(A \rightarrow C))$

(d) $(C \rightarrow(A \rightarrow B)) \rightarrow((C \rightarrow A) \rightarrow(C \rightarrow(C \rightarrow B)))$

Applying (c) and (d) to (b) and using A2 we have $D \rightarrow((C \rightarrow A) \rightarrow(C \rightarrow(C \rightarrow$ $B))$ ), and then $(C \rightarrow A) \rightarrow(C \rightarrow(C \rightarrow(D \rightarrow B)))$. Therefore, using (a), $C \rightarrow$ $(C \rightarrow(D \rightarrow B))$, i.e., $B_{(1,2,3)}^{*}$ is a theorem of $\mathbf{P}$.

Thus one can see that the disjointness requirement in $\mathbf{R} 2$ derives from the fact that (d) is a theorem of $\mathrm{P}$ but $(C \rightarrow(A \rightarrow B)) \rightarrow((C \rightarrow A) \rightarrow(C \rightarrow B))$ is not: $Q$ may of course be extended by adding rules for negation, disjunction, and the quantifiers.

\section{REFERENCES}

[1] Ackermann, Robert, An Introduction to Many-Valued Logics, Dover Publications, Inc., New York (1967), p. 50.

[2] Suppes, Patrick, Introduction to Logic, Van Nostrand, Princeton (1957).

[3] Woodruff, Peter W., "Logic and Truth Value Gaps," Philosophical Problems in Logic (ed. by K. Lambert), D. Reidel Publishing Company, Dordrecht (1970). 